\title{
Far-field power transmissions in orthotropic plates: A new approach
}

\author{
Nirmal K. Mandal \\ Centre for Railway Engineering, Central Queensland University, Bruce Highway, North Rockhampton, Queensland \\ 4702, Australia \\ E-mail: n.mandal@cqu.edu.au
}

Received 8 March 2004

Revised 20 April 2006

\begin{abstract}
The structural intensity (SI) technique is an essential tool for locating and ranking vibration sources and sinks on structures. It can quantify vibration fields by plotting a vector map of energy transmission on the structures. In this paper, a different strategy, changing coordinate systems of plate equations, is used to develop an intensity equation from shear force components in both $x$ and $y$ directions. The formulation is carried out in the frequency domain considering flexural waves. Orthotropic plate theory, far-field conditions, Fourier transform, and finite difference approximation are considered. The same intensity definition is obtained using this different strategy. A dual-channel FFT analyser is essential for data acquisition to get an intensity vector in a particular direction for far-field conditions.
\end{abstract}

\section{Introduction}

The SI or vibration intensity (VI) technique is now considered to be a significant measurement tool for structureborne sound. It yields not only the information of locations of vibration sources and sinks, but also estimates the reflection coefficients (edge effects), and mechanical impedances of the structures. On the other hand, it can be effectively employed to identify the propagation paths of vibration energy transmission. This is possible by plotting $\mathrm{x}$ - and $\mathrm{y}$ - components of intensity vector at a number of points on the structures. As a result, proper damping treatment can be applied to the area of energy transfer. Thus, it can be widely used in industrial application for controlling noise and vibration harness $(\mathrm{NVH})$ problems either by surface damping treatment or by isolation of energy sources from the structures.

The SI technique was developed in the early 1970's. Most of the previous methods using SI are useful for simple structures, typically beams and thin plates in flexure [1-6]. Some of these formulations are in the time domain [2] and others are in the frequency domain [3,4]. Non-contact methods using acoustic holography [7-10] and optical measurements [11-14] are also available. Numerical analysis using finite element approach is a good alternative [15, 16]. Recently, SI has been employed for flexural waves in complex structures such as in orthotropic plates for farfield conditions [17] as an approximate method, and in general field conditions [18] as an exact method. It requires acquisition of all field signals at the same time and ensemble averaging should be performed. Proper attention should be provided in instrumentation so as to minimize measurement errors. Experimental studies of energy transmission in corrugated plates have been carried out recently considering flexural waves [23] and in-plane waves [24].

It is observed, through the literature review, that few researches had been undertaken on orthotropic plates using SI. Orthotropic plates such as corrugated plates, beam-stiffened plates, and plate-grid structures are the most important components in industry especially in automobiles, aircraft structures, ship structures, railway bogies and decking and machine casings. It is of the utmost importance to control noise and vibration of such structures. It is, therefore, necessary to get a useful measurement method for those structures. In this paper, VI is used to develop an intensity formulation for flexural waves applicable to thin naturally orthotropic plates from shear force component only. Through this article, a preliminary result of shear force contribution to total power will be focused on. An analysis of individual contribution to energy transfer from shear force and moments is sometimes useful [4]. Later, both contributions from shear force and moments will be taken into account to obtain total power. 


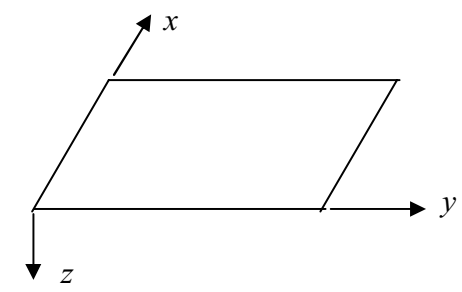

Fig. 1. Co-ordinate system of naturally orthotropic plate.

\section{Theoretical analysis}

\subsection{Orthotropic plate equation}

This study considers a thin homogeneous orthotropic plate (Fig. 1) with small deflections compared to the uniform thickness. By definition, a thin plate results when the thickness of the plate, $h$, is small enough compared to other dimensions. In thin plate flexural wave equation, the influences of rotary inertia and shear deformation are neglected. This approximation is valid when $h \ll \lambda$, the flexural wavelength [19].

The analysis of classical orthotropic plate theory can be found in many literatures $[19,20]$. The orthotropic plate equation for free, harmonic, time dependent flexural vibration can be obtained as [18],

$$
\left(D_{x} \frac{\partial^{4} w}{\partial x^{4}}+2 H \frac{\partial^{4} w}{\partial x^{2} \partial y^{2}}+D_{y} \frac{\partial^{4} w}{\partial y^{4}}\right)=m^{\prime \prime} \omega^{2} w
$$

Where, $\omega$ is the angular frequency, $m^{\prime \prime}$ is the mass per unit area of the plate, $D_{x}$ and $D_{y}$ are called the flexural rigidities, $w$ is the transverse displacement of the plate, and $2 H$ is called the effective torsional rigidity of the orthotropic plate (Eq. (2)), and $w$ is the transverse deflection in $z$-direction.

$$
H=D_{x} \nu_{y}+2 G_{x y}
$$

where $G_{x y}$ is the shear modulus of elasticity and $\nu_{y}$ is the Poission's ratio. Due to the complexity of analysis with regard to $H$, many researchers in the area of orthotropic plates consider an approximation of $H$ such as $H=\sqrt{D_{x} D_{y}}$ in orthotropic plate equations. This approximation gives accurate results for the analysis of orthotropic plates [19]. In this case, if the coordinate system changes to a new system such that $x$ is unchanged and $y$ is changed to $y^{\prime}=y\left(D_{x} / D_{y}\right)^{1 / 4}$, the flexural wave equation for orthotropic plates results in the same form as that for isotropic plates [19]. Consequently, an exact solution of an orthotropic plate problem is possible. This modified coordinate for the plate (Fig. 1) takes another system of $\left(x, y^{\prime}\right)$. In the following section, this idea is used to modify the orthotropic plate Eq. (1) to obtain a far-field wave equation.

\subsection{Modified plate equation}

In an earlier analysis [17], authors used dimensionless parameters to model the orthotropic plate equation for approximate far-field formulation. Both $x$ and $y$ coordinates were transformed to dimensionless parameters. In this case, only the $y$ coordinate is modified which is not dimensionless. As the plate flexural deformation depends on both $x$ and $y^{\prime}$ coordinates, the related terms in Eq. (1) can be obtained by partial differentiation with respect to $y^{\prime}$.

$$
\begin{aligned}
& \frac{\partial w}{\partial y}=\frac{\partial w}{\partial y^{\prime}}\left(\frac{D_{x}}{D_{y}}\right)^{1 / 4} \\
& \frac{\partial^{2} w}{\partial y^{2}}=\frac{\partial^{2} w}{\partial y^{\prime 2}}\left(\frac{D_{x}}{D_{y}}\right)^{1 / 2} \\
& \frac{\partial^{4} w}{\partial y^{4}}=\frac{\partial^{4} w}{\partial y^{\prime 4}}\left(\frac{D_{x}}{D_{y}}\right)
\end{aligned}
$$


Using Eqs (3a, b, c) in Eq. (1), it is possible to obtain the orthotropic plate equation incorporating the new coordinate system $\left(x, y^{\prime}\right)$.

$$
\left(\frac{\partial^{4} w}{\partial x^{4}}+2 \frac{\partial^{4} w}{\partial x^{2} \partial y^{\prime 2}}+\frac{\partial^{4} w}{\partial y^{\prime 4}}\right)=\frac{m^{\prime \prime} \omega^{2}}{D_{x}} w
$$

This Eq. (4) behaves like the isotropic plate equation. If $k$ is flexural wave number such that $k^{4}=\frac{m^{\prime \prime} \omega^{2}}{D_{x}}$, the above plate equation may take a new factorized form as

$$
\left(\frac{\partial^{2}}{\partial x^{2}}+\frac{\partial^{2}}{\partial y^{\prime 2}}\right)^{2} w=k^{4} w
$$

The bracketed term may be denoted by $\Delta$ (such that $\Delta=\frac{\partial^{2}}{\partial x^{2}}+\frac{\partial^{2}}{\partial y^{\prime 2}}$ ), the Laplace operator. Although a different transformation is considered in this analysis, the definition of flexural wave number, $k$, is same as in [17] but $\Delta$ takes a new form. Further simplification of Eq. (5) is possible and can be transformed as

$$
\left(\Delta+k^{2}\right)\left(\Delta-k^{2}\right) w=0
$$

The Eq. (6) can be transferred to two equations as

$$
\begin{aligned}
& \left(\Delta+k^{2}\right) w=0 \\
& \left(\Delta-k^{2}\right) w=0
\end{aligned}
$$

Equation (7a) represents the condition of far-field where a free propagating wave exists. A complete solution of this equation may be possible. The Eq. (7b), on the other hand, is the condition of near-field [19] as the disturbances decay exponentially from sources and boundaries. In the following section, the idea of far-field plate Eq. (7a) is used to modify the shear force relation useful for orthotropic plates.

\subsection{Modified shear force equation in the far-field conditions}

The equation of shear force in $x$-direction of an orthotropic plate (Fig. 1) can be obtained [20] as

$$
Q_{x}=-\frac{\partial}{\partial x}\left(D_{x} \frac{\partial^{2} w}{\partial x^{2}}+H \frac{\partial^{2} w}{\partial y^{2}}\right)
$$

Again, incorporating the new coordinate system $\left(x, y^{\prime}\right)$, the shear force Eq. (8) may change to another form as

$$
\begin{aligned}
Q_{x} & =-D_{x} \frac{\partial}{\partial x}\left(\frac{\partial^{2} w}{\partial x^{2}}+\frac{\partial^{2} w}{\partial y^{\prime 2}}\right) \\
& =k^{2} D_{x} \frac{\partial w}{\partial x}
\end{aligned}
$$

The Eq. (9b) is the equation of shear force for the condition of far-field.

Far-field equation for shear force in the $y$-direction can be obtained in a similar manner. The equation of shear force in $y$-direction can be presented in terms of spatial derivatives with $D_{y}$ and $H$ as [20]

$$
Q_{y}=-\frac{\partial}{\partial y}\left(D_{y} \frac{\partial^{2} w}{\partial y^{2}}+H \frac{\partial^{2} w}{\partial x^{2}}\right)
$$

If a new coordinate system is incorporated in Eq. (10), the shear force takes a different form with modified spatial derivatives as

$$
Q_{y}=-\sqrt[4]{D_{x}^{3} D_{y}} \frac{\partial}{\partial y^{\prime}}\left(\frac{\partial^{2} w}{\partial y^{\prime 2}}+\frac{\partial^{2} w}{\partial x^{2}}\right)
$$




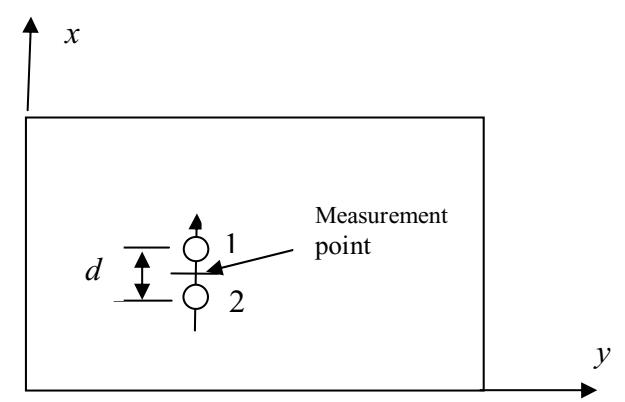

Fig. 2. Two-transducer array, measuring vibration power transmission from point 2 to point 1 a distance $d$ apart.

Putting the condition of far-field, Eq. (7a), in Eq. (11), it is possible to obtain the far-field component of shear force in $y$-direction as

$$
Q_{y}=\sqrt[4]{D_{x}^{3} D_{y}} k^{2} \frac{\partial w}{\partial y^{\prime}}
$$

If the coordinate system changes back to $(x, y)$ using Eq. (3a), and putting in the value of wave number, $k$, it is possible to re-formulate the Eq. (12) into a new form as

$$
Q_{y}=\omega \sqrt{m^{\prime \prime} D_{y}} \frac{\partial w}{\partial y}
$$

If the value of wave number is substituted into the Eq. (9b), a similar equation that of Eq. (13) can be obtained. It is the far-field equation of shear force in $y$-direction.

In the following section, shear force power is formulated first considering the component in the $x$-direction and a similar method is applied to obtain SI in the $y$-direction.

\section{Shear force power}

It is a common practice to use FFT analyzer for the detection of power flow in structures. Multiple signals can be accommodated in analyzer and analysis is performed in the frequency domain, which replaces the time averaging steps of power flow formulation in time domain. In the frequency domain, the complex power from shear force is the cross-spectrum of velocity and force component and is given by the following relation

$$
P_{x S}(f)=G_{v F}(f)
$$

where $P_{x s}(f)$ is the complex power in $x$-direction of the plate, and $G_{v F}(f)$ is the cross-spectrum of transverse velocity and shear force.

Differentiating the Eq. (9b) with respect to time and taking Fourier transform, it is possible to get the $x$-component of shear force equation in terms of transverse velocity as

$$
\hat{Q}=\frac{D_{x} k^{2}}{j \omega} \frac{\partial \hat{v}}{\partial x}
$$

The shear force components in Eqs (9a) and (13) are based on transverse displacement, w. The spatial derivative of shear force Eq. (15) can be obtained using finite difference approximation (Fig. 2). The $x$-component of SI from shear force can thus be obtained as

$$
P_{x S}=<-\hat{v}^{*} \hat{Q}_{x}>
$$

where $<>$ represents ensemble average. The cross-spectrum of the Eq. (14) can be defined as in Eq. (16) [21]. The negative sign is included to make power flow positive in positive direction [2].

Using finite difference approximation, the transverse velocity and spatial derivatives of transverse velocity in Eqs (15) and (16) can be obtained as (Fig. 2) 


$$
\begin{aligned}
& \hat{v}=\frac{\hat{v}_{1}+\hat{v}_{2}}{2} \\
& \frac{\partial \hat{v}}{\partial x}=\frac{\hat{v}_{1}-\hat{v}_{2}}{d}
\end{aligned}
$$

Hence, the complex far-field power from shear force in $x$-direction of the plate is as follows

$$
P_{x s}=<-\left(\frac{\hat{v}_{1}+\hat{v}_{2}}{2}\right)^{*} \frac{D_{x} k^{2}}{j \omega}\left(\frac{\hat{v}_{1}-\hat{v}_{2}}{d}\right)>
$$

Evaluating the ensemble averages term by term, the final complex form of structural intensity in the $\mathrm{x}$-direction by shear force only would be obtained as

$$
P_{x S}(f)=\frac{D_{x} k^{2}}{2 j \omega d}\left[\left(G_{22}-G_{11}\right)+2 j \operatorname{Im}\left\{G_{12}\right\}\right]
$$

where $d$ is the distance between two successive points, $\omega$ is the angular frequency, $G_{12}$ is the cross-spectrum of the velocity signals at points 1 and 2 and $G_{22}$ and $G_{11}$ are two auto-spectrum of the velocity signals. The real part of the above complex power flow Eq. (19) presents the power transmitting in the far-field of the orthotropic plates by shear force in the $x$-direction and it could be written as

$$
I_{x}(f)=\frac{\sqrt{D_{x} m^{\prime \prime}}}{d} \operatorname{Im}\left\{G_{12}\right\}
$$

In the above equation, $I$ is used to represent $S I$ because it is active component of complex power and to differentiate it from the complex power, $P$. Similarly, the $y$-component of shear force considering cross-spectrum of velocity signal can be derived as

$$
I_{y}(f)=\frac{\sqrt{D_{y} m^{\prime \prime}}}{d} \operatorname{Im}\left\{G_{12}\right\}
$$

Since accelerometers are widely used in vibration measurements, it is therefore customary to use acceleration signals instead of velocity signals in the formulation of vibration power.

In the frequency domain, the relation between velocity and acceleration is $\hat{v}=\frac{\hat{a}}{j \omega}$. This leads to $G_{v v}=\frac{G_{a a}}{\omega^{2}}$ for power spectral densities of acceleration and velocity where $\hat{v}$ and $\hat{a}$ are the signals of velocity and acceleration in the frequency domain respectively. The following cross-spectrum $G_{i j}$ refer to the signals of acceleration rather than velocity. By using the acceleration based cross-spectrum, the structural intensity Eq. (16) could be rewritten as

$$
I_{x}(f)=\frac{\sqrt{D_{x} m^{\prime \prime}}}{d \omega^{2}} \operatorname{Im}\left\{G_{12}\right\}
$$

The orthogonal component ( $y$ component) of structural intensity from shear force can thus be achieved by exchanging the letter $x$ to $y$.

$$
I_{y}(f)=\frac{\sqrt{D_{y} m^{\prime \prime}}}{d \omega^{2}} \operatorname{Im}\left\{G_{12}\right\}
$$

\section{Discussions}

In this article, cross-spectrum density function is used to formulate far-field power flow equation for orthotropic plates in $x$ and $y$ directions from shear force in the frequency domain. A new approach is used to get the same relation as that was obtained before [17]. By changing the orthotropic plate equation to a form like that of isotropic plate, the solution converges to be exact [19]. A dual channel FFT analyzer may be used to take simultaneous acquisition of field data. Careful considerations should be given for instrumentation to reduce measurement error during data acquisition. There are two types of inherent error: one is due to finite difference and the other is due to phase mismatch of transducers. 
Coefficients of spatial derivatives in orthotropic plate equation in bending are different from those for isotropic plate. These derivatives are: $D_{x}, D_{y}$ and $H$ (Eq. (1)). Only $D$ applies for the case of isotropic plate. This enables the modification of isotropic plate equation to provide usable relations to solve practical applications in industry for NVH problems. This is not possible directly in the case of orthotropic plates. As a result, researchers and engineers working in this area used some assumptions such as $\left(H=\sqrt{D_{x} D_{y}}\right)[17,19,20,23]$ and obtained accurate results. As orthotropic plates such as rectangular, trapezoidal and sinusoidal corrugated plates and beam stiffened plates are widely used in industries, it is necessary to simplify the theoretical formulations into useable equations for FFT analyser's usage. Therefore it yields a significant advantage in providing solutions with small errors in NVH problems.

As is stated above, the flexural wave equation in orthotropic plate is completely different to that of isotropic plate. In the latter case, flexural rigidity $(D)$ comes out from each spatial term of the equations of shear force and bending and twisting moments as a common factor. As a result, it is very simple to replace far-field wave condition in shear force and modified moment relations [1]. This is not directly possible for orthotropic plates because of different rigidity values in their spatial terms such as $D_{x}, D_{y}$ and $H$. A problem for shear force component is solved here and currently work is being continued for the solution of moment part. When it is solved, it will be put forward as an extension of this paper for total power from both shear force and moments to remove few assumptions.

The difference between the present analysis and that of ref. [17] is evident. In the present analysis, only $y$ coordinate is changed to $y^{\prime}$ incorporating rigidity ratio $\left\{y^{\prime}=y\left(D_{x} / D_{y}\right)^{1 / 4}\right\}$. By comparison, in the analysis of ref. [17], both $x$ and $y$ coordinates are transferred to dimensionless parameters incorporating the plate dimensions of $\mathrm{a}$ and $\mathrm{b}$ as follows: $\xi=\frac{x}{a}, \eta=\frac{y}{b}$ and $\gamma_{o r t}=\frac{a}{b} \sqrt[4]{\frac{D_{y}}{D_{x}}}$. This results in a different strategy in the work stated in ref. [17].

Averaging in the time domain and the frequency domain differs. In the time domain, few data are added and divided by the period of time $\left\{\overline{x(t)}=\lim _{T \rightarrow \infty} \frac{1}{T} \int_{-T / 2}^{T / 2} x(t) d t\right\}$. In the frequency domain, on the other hand, averaging is called an ensemble average. In this type, sample data are taken for a specific time at transducer locations and an average is obtained dividing by the number of samples $\left\{\left\langle x\left(t_{1}\right)\right\rangle=\lim _{N \rightarrow \infty} \frac{1}{N} \sum_{i=1}^{N} x^{(i)}\left(t_{1}\right)\right\}$, where $N$ is number of samples. In other words, it is the expected operation over a collection of raw finite Fourier transforms. This expected operation is called ensemble average. In FFT analyzer, it is possible to format its setting to any average such as 200 or 300 , and the analyzer performs the expected operations. However, last state of a sample may not match with the first state of next sample. As a result, some errors occur, called leakage. Hanning window may be employed to provide best solution relating to the leakage. FFT analyzer can perform time domain average too. This average ensures an accuracy of results. A high value of coherence function (close to unity) between input signal (force) and output signal (acceleration) yields a result to be trusted. This high value of coherence function can be achieved using a larger number of averages taken during data acquisition. Although the expected operations of cross-spectra of Eq. (12) are carried out in the frequency domain, it yields a time average net power [21]. This is an established technique in the area of signal processing. Analysis in time domain and frequency domain has relative advantages and disadvantages. It is recently discussed by Mandal et al. [22].

Vector plot of SI represents its magnitude and direction at a point (resultant of $x$ and $y$ components). From source, vibration power is flowing out, meaning that all intensity vectors are going out from a point (location of attached electrical motor, for example). Sink (viscous damper for example), on the other hand, absorbs energy. In this location SI vectors flow to this point. Therefore a vector map shows the location of sources and sinks. The magnitude of SI can be presented by its numerical or $\mathrm{dB}$ values, indicating its ranking. The propagation paths can also be identified from this plot.

SI is obtained from the cross-spectra of force and velocity signals. Displacement (deformation), velocity and acceleration are interrelated. The magnitude and phase of SI are dependent on deformation. In the case of harmonic vibration for example, magnitude and phase of deformation are changing from location to location. Therefore it has a significant influence on SI.

These models (Eqs (22) and (23)) are applicable for orthotropic plates with uniform thickness. The applicability of uniform orthotropic plate theory in rib-plates, and corrugated plates depends on the flexural wavelength. It is established that the flexural wavelength of these plates should be considerable greater than one repeating section of rib or corrugation [19]. This is very important in high frequency range when the associated wavelength is shorter. By 
using filtering technique in data acquisition, it is possible to remove the frequency range where wavelength may not be considerably greater than the distance of one repeating section. Alternately the idea of elastic equivalence [20] can be useful to model technically orthotropic plates such as corrugated plates, ribbed plate, plate grids, beam reinforced plates and similar other plates. This technique transfers technically orthotropic plate to naturally orthotropic plate of uniform thickness [20]. Therefore this model can be easily applied using FFT analyzer to estimate vibration power flow in industrial applications.

\section{Conclusions}

A two-transducer technique for SI calculation is proposed considering a different approach. A Change in the coordinate system enables the researchers to obtain flexural wave equations of plate to a simpler form to get a far-field condition. This SI formulation is from shear force only for both $x$ and $y$ directions incorporating a cross-spectrum method. The two transducer model helps to use FFT analyzer for practical data acquisition in the frequency domain. For complete analysis, it is necessary to consider the contribution of moments. However, at this stage, it is put forward as a preliminary result. In a separate article, the contribution both from shear force and moments will be considered later using the $x, y^{\prime}$ coordinate.

\section{References}

[1] D.U. Noiseux, Measurement of power flow in uniform beams and plates, Journal Acoustical Society of America 47(1) (1970), $238-247$.

[2] G. Pavic, Measurement of structure borne wave intensity, part I: Formulation of the methods, Journal of Sound Vibration 49(2) (1976), 221-230.

[3] J.W. Verheij, Cross-spectral density methods for measuring structure borne power flow on beams and pipes, Journal of Sound Vibration 70(1) (1980), 133-138

[4] J. Linjama and T. Lahti, Estimation of bending wave intensity in beams using the frequency response technique, Journal of Sound Vibration 153(1) (1992), 21-36.

[5] P.D. Bauman, Analytical and experimental evaluation of various techniques for the case of flexural waves in one-dimensional structures, Journal of Sound Vibration 174(5) (1994), 677-694.

[6] J.R.F. Arruda and J.P. Campos, Experimental determination of flexural power flow in beams using a modified prony method, Journal of Sound Vibration 197(3) (1996), 309-328

[7] E.G. Williams and H.G. Dardy, Near field acoustic holography using an underwater-automated scanner, Journal Acoustical Society of America 78 (1985), 789-798

[8] J.D. Maynard, E.G. Williams and Y. Lee, Near field acoustic holography: I. Theory of generalised holography and development of NAH, Journal Acoustical Society of America 78 (1985), 1395-1413.

[9] E.G. Williams, H.D. Dardy and R.G. Fink, A technique for measurement of structure-borne intensity in plates, Journal Acoustical Society of America 78 (1985), 2061-2968.

[10] A.J. Romano and E.G. Williams, On the use of acoustic holography for the determination of intensity of structures, Proceeding of the 4 th International Congress on Intensity Technique, Senlis, France, 1993, 13-34.

[11] J. Linjama, Structural intensity measurements using two laser vibrometers, Inter-noise 92 (1992), 541-544

[12] J.C. Pascal, T. Loyau and X. Carniel, Complete determination of structural intensity in plates using laser vibrometers, Journal of Sound Vibration 161(3) (1993), 527-531.

[13] T.E. McDevitt, G.H. Koopmann and C.B. Burroughs, Two-channel laser vibrometer Techniques for vibration intensity measurements, Part 1: Flexural intensity, Journal of Vibration and Acoustics 115 (1993), 436-440.

[14] Y.H. Berthelot, M. Yang and J. Jarzynski, Recent progress on laser doppler measurement in structural acoustics, Proceeding of the $4^{h}$ International Congress on Intensity Technique, Senlis, France, 1993, 199-206.

[15] L. Gavric and G. Pavic, A finite element method for computation of structural intensity by the normal mode approach, Journal of Sound Vibration 164(1) (1993), 29-43.

[16] S.A. Hambric and P.D. Taylor, Comparison of experimental and finite element structure-borne flexural power measurements for a straight beam, Journal of Sound Vibration 170 (1994), 595-605.

[17] N.K. Mandal, R. Abd. Rahman and M.S. Leong, Prediction of Structure borne sound in orthotropic plates for far field conditions, Journal of Vibration and Control 8(1) (2002), 3-12.

[18] N.K. Mandal, R. Abd. Rahman and M.S. Leong, Structure-borne power transmission in thin naturally orthotropic plates: General Case, Journal of Vibration and Control 9(10) (2003), 1189-1199.

[19] L. Cremer and M. Heckl, Structure-Borne Sound: Structural Vibration and Sound Radiation at Audio Frequencies, Springer-Verlag, Berlin, 1988.

[20] M.S. Troitsky, Stiffened Plates: bending, stability and vibration, Amsterdam: Elsevier Scientific Publishing Company, 1976.

[21] J.S. Bandat and A.G. Piersol, Random Data: Analysis and Measurement Procedures, New York: John Wiley, $2^{\text {nd }}$ edition, 1986. 
[22] N.K. Mandal and S. Biswas, Vibration Power Flow: A Critical Review, The Shock and Vibration Digest 37(1) (2005), 3-11.

[23] N.K. Mandal, R. Abd. Rahman and M.S. Leong, Experimental Investigation of Vibration Power Flow in Thin Technical Orthotropic Plates by the Method of Vibration Intensity, Journal of Sound Vibration 285(3) (2005), 669-695.

[24] N.K. Mandal, Experimental studies of quasi-longitudinal waves power flow in corrugated plates, Journal of Sound Vibration 297 (2006), $227-242$. 

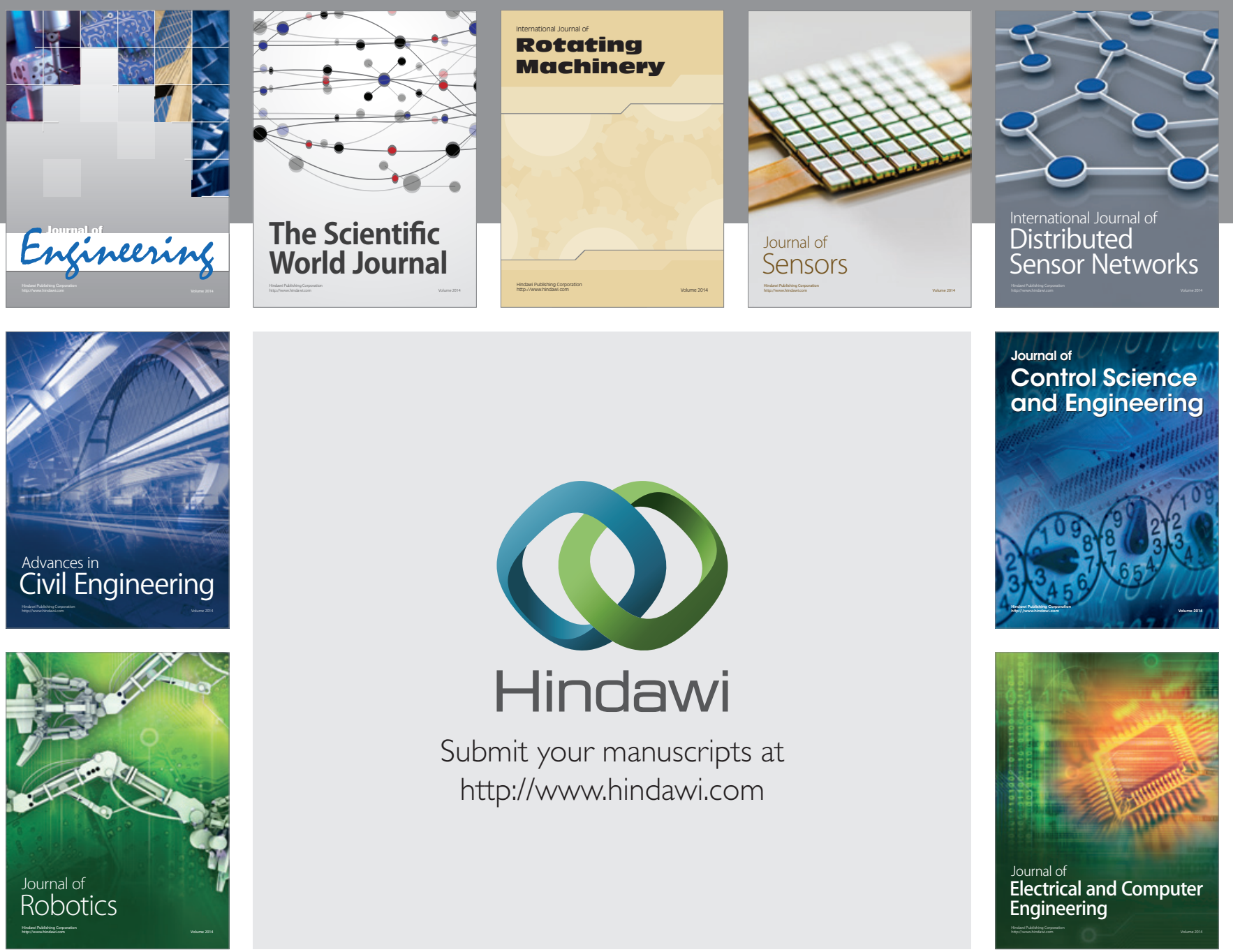

Submit your manuscripts at

http://www.hindawi.com
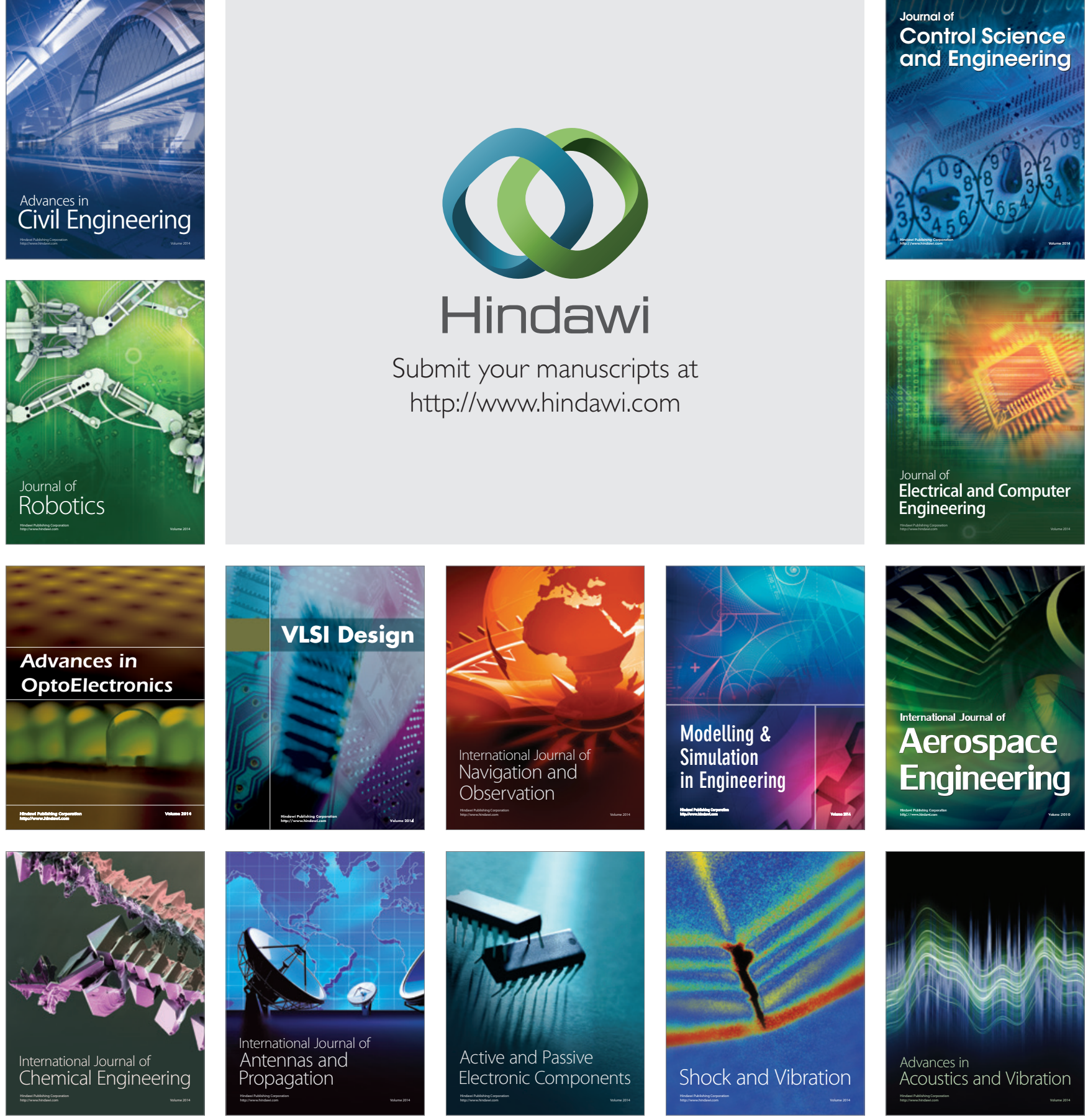